\title{
BMJ Open Exploration of prescribing error reporting across primary care: a qualitative study
}

\author{
Nicola Hall (D) , ${ }^{1,2}$ Kathryn Bullen, ${ }^{3}$ John Sherwood, ${ }^{3}$ Nicola Wake, ${ }^{4,5}$ Scott Wilkes, ${ }^{3}$ \\ Gemma Donovan ${ }^{2}$
}

To cite: Hall N, Bullen K, Sherwood J, et al. Exploration of prescribing error reporting across primary care: a qualitative study. BMJ Open 2022;12:e050283. doi:10.1136/ bmjopen-2021-050283

- Prepublication history for this paper is available online. To view these files, please visit the journal online (http://dx.doi. org/10.1136/bmjopen-2021 050283).

Received 16 February 2021 Accepted 07 December 2021

D) Check for updates

(C) Author(s) (or their employer(s)) 2022. Re-use permitted under CC BY-NC. No commercial re-use. See rights and permissions. Published by BMJ.

${ }^{1}$ Population Health Sciences Institute, Faculty of Medical Sciences, Newcastle University, Newcastle upon Tyne, UK ${ }^{2}$ Faculty of Health Sciences and Wellbeing, University of Sunderland, Sunderland, UK ${ }^{3}$ School of Pharmacy, University of Sunderland, Sunderland, UK ${ }^{4}$ Northumbria Healthcare NHS Foundation Trust, North Shields, UK

${ }^{5}$ NHS Specialist Pharmacy

Service, London North West Healthcare NHS Trust Pharmacy Service, Harrow, UK

Correspondence to

Dr Nicola Hall;

nicola.hall@newcastle.ac.uk

\section{ABSTRACT}

Objectives To explore barriers and facilitators to prescribing error reporting across primary care. Design Qualitative semi-structured face-to-face and telephone interviews were conducted to explore facilitators and barriers to reporting prescribing errors. Data collection and thematic analysis were informed by the COM-B model of behaviour change. Framework analysis was used for coding and charting the data with the assistance of NVivo software (V.12). General and context specific influences on prescribing error reporting were mapped to constructs from the COM-B model (ie, capability, opportunity and motivation).

Setting Primary care organisations, including community pharmacy, general practice and community care from North East England.

Participants We interviewed a maximal variation purposive sample of 25 participants, including prescribers, community pharmacists and key stakeholders with primary care or medicines safety roles at local, regional and national levels.

Results Our findings describe a range of factors that influence the capability, opportunity and motivation to report prescribing errors in primary care. Three key contextual factors are also highlighted that were found to underpin many of the behavioural influences on reporting in this setting: the nature of prescribing; heterogeneous priorities for error reporting across and within different primary care organisations; and the complex infrastructure of reporting and learning pathways across primary care. Findings suggest that there is a lack of consistency in how, when and by whom, prescribing errors are reported across primary care.

Conclusions Further research is needed to identify cross-organisational and interprofessional consensus on agreed reporting thresholds and how best to facilitate a more collaborative approach to reporting and learning, that is, sensitive to the needs and priorities of disparate organisations across primary care. Despite acknowledged challenges, there may be potential for an increased role of community pharmacy in prescribing error reporting to support future learning.

\section{BACKGROUND}

Medication errors are a leading cause of injury and avoidable harm in healthcare systems across the world and have been estimated to

\section{Strengths and limitations of this study}

- A key strength of this study is the inclusion of a wide range of information-rich perspectives from prescribers, pharmacists and other key stakeholders with local, regional and national roles in medicines optimisation and safety.

- Findings were informed but not constrained by the COM-B model of behaviour which provided a valuable theoretical lens to help identify and categorise a range of influences on the capability, opportunity and motivation to report prescribing errors across the primary care setting.

- Data collection and analysis continued until thematic saturation was reached, strengthening confidence in our findings.

- We were not able to include key perspectives from prescribers or community pharmacists from a wider geographical area, from stakeholders from relevant regulatory bodies, dentists or prescribing staff from community nursing and transferability to other contexts may therefore be limited.

- Participants who agreed to be interviewed are likely to have had a specific interest in, and positive attitudes towards, the value of prescribing error reporting and learning.

cost US $\$ 42$ billion annually. ${ }^{12}$ The WHO's third global challenge, launched in $2017,^{2}$ aims to reduce the level of severe, avoidable harm related to medications globally by $50 \%$ over 5 years. ${ }^{3}$ This is also a key government priority in the UK. ${ }^{4}$

In England, it has been estimated that 237.4 million medication errors occur every year with $25.8 \%$ and $2.0 \%$ of overall errors having the potential to cause moderate or severe harm, respectively. ${ }^{5}$ The importance of supporting a culture that encourages the reporting of medication errors is widely recognised as one of the ways in which to identify patterns, promote shared learning and reduce medication-related harm. ${ }^{67}$ In the UK, systems learning based around medical error reporting are supported by a number 
of policy and technological initiatives. ${ }^{8}$ The UK's national electronic reporting system (the National Reporting and Learning System (NRLS)) is currently being updated with a new Patient Safety Incident Management System (PSIMS) ${ }^{9}$ by National Health Service (NHS) England and NHS Improvement who have taken over the responsibilities from the former National Patient Safety Agency. ${ }^{10}$ Other initiatives include a National Medication Safety network $^{8}$ and within primary care, recommendations for significant event analysis to be undertaken regularly by primary care teams ${ }^{411}$ in line with Care Quality Commission standards around ensuring lessons are learnt when things go wrong. ${ }^{12}$

Although the focus of research on error reporting has been mostly based within hospital care, there has been an increasing attention on primary care settings, where under-reporting of medication errors is reported to be common. ${ }^{13}$ Due to the volume of patients seen in primary care, this setting is where most prescriptions are generated within the health system. ${ }^{14}{ }^{15}$ It has been estimated that up to $70 \%$ of medication errors within primary care are prescribing errors, ${ }^{16}$ and an audit in general practices in England identified an error rate of $12.5 \%$ in medicines prescribing. ${ }^{17}$ Furthermore, around $5 \%$ of prescriptions in primary care are estimated to include suboptimal or unsafe prescribing, although this figure varies widely and definitions are used inconsistently throughout the literature. ${ }^{18} 19$

Prescribing errors originating from general practice and other community health services are often identified and rectified within community pharmacy, as pharmacists clinically check prescriptions for appropriateness before dispensing medicines to patients. ${ }^{520}$ These checks have been reported to make a significant cost contribution to the healthcare economy as part of avoided patient harm. ${ }^{52}$ Although patient safety incident analysis is currently being brought together in England across local health economies under new arrangements for co-commissioning of general practice between NHS England and Clinical Commissioning Groups (CCGs), ${ }^{22}$ general practitioners (GPs) and community pharmacy act as independent contractors, and organisational structures within the NHS do not always support feedback and learning across these boundaries.

Decisions and behaviours around medication error reporting can be complex, ${ }^{18}$ and a number of barriers to error reporting have been identified across other settings and error types. These include: awareness and functionality of incident reporting systems; ${ }^{23} 24$ the need to clarify reporting criteria; ${ }^{25}$ and sociocultural, organisational and political processes and agendas relating to professional practice, regulation, social inequalities, control and power. ${ }^{26-28}$ Personal thresholds for reporting have also been shown to vary within and between health professions, ${ }^{29}{ }^{30}$ and evidence suggests that medication error reporting rates can improve with intervention, ${ }^{31} 32$ although sustainability beyond trial settings is less clear.
There is currently little evidence on the context specific influences on prescribing error reporting behaviour across primary care ${ }^{1433}$ and no study that we are aware of has explored the influences on prescribing error reporting within the primary care system as a 'whole'. The aim of this study was therefore to explore key facilitators and barriers to prescribing error reporting across primary care. A particular focus was placed on exploring the role, or potential role, of community pharmacy due to its key responsibilities in the identification and rectification of medication errors within primary care. ${ }^{621} 22$

\section{METHODS}

\section{Study design}

This was a qualitative study using semi-structured interviews with participants from primary care organisations, including GPs, pharmacists and other stakeholders with key roles in medication safety and prescribing quality in primary care.

\section{Setting and sample}

The study was undertaken in a range of primary care organisations within North East England. We purposively recruited a maximal variation sample of prescribers from: general practice and community health services (ie, GPs, nurse practitioners, including those from out of hours services); community pharmacy professionals from a range of independent and national chain pharmacies (ie, pharmacists, prescribing pharmacists); and other key stakeholders (ie, commissioners, medication and prescribing safety leads, superintendent pharmacists, directors of nursing and, or, safety). Our sampling strategy aimed to gain a wide range of perspectives from across primary care, including those with different medication prescribing, optimisation and safety roles at the local, regional and national level. The aim was to enable a greater understanding of the influences on prescribing error reporting behaviours across the multiorganisational primary care setting as a 'whole'.

Recruitment sources used to help identify potential participants included professional bodies and networks (Royal College of General Practitioners, the North East Sessional GPs group, medication or patient safety groups, local pharmaceutical committees, local and national community pharmacy organisations and regulatory bodies), the North East Commissioning Support Unit and local and regional commissioning organisations. Potential participants were also contacted directly via the research team's professional networks, as well as through wider promotional activities, such as electronic newsletters and mailings and social media. Those interested in taking part were asked to contact the study researcher directly. Informed consent was gained by all participants.

\section{Data collection}

Semi-structured interviews were conducted face-to-face either at the university where the researcher was based 
or at the place of work of the interviewee. If this was not possible, interviews were completed by telephone. They allowed an in-depth exploration of views on what constitutes a reportable prescribing error, the identification of key facilitators and barriers to prescribing error reporting within primary care, the extent current crossorganisational reporting and learning systems influence reporting, and perceived gaps in prescribing error intelligence within each type of organisation. Topic guides were informed by the COM-B model of behaviour, ${ }^{34}$ based on evidence that a person is more likely to enact a behaviour when they have the capability and opportunity to engage in the behaviour and there is a greater motivation to enact that behaviour than any others. COM-B was chosen as an appropriate theoretical framework to help understand the influences on prescribing error reporting as a behaviour as well potential strategies for behaviour change going forward, in light of evidence suggesting that prescribing errors are likely to be under-reported ${ }^{10} 14$ and findings based on reporting behaviour from other settings. ${ }^{30-32}$ This model is an interactional model which has been widely applied in health research. It proposes that capability and opportunity influence motivation, that all three influence behaviour change but are also influenced by the change which occurs. The model was used as a 'theoretical lens' to guide data collection and analysis, allowing a theory-informed understanding of the influences on prescribing error reporting. However, participant-led topics were also encouraged and pursued in a more inductive manner during the interviews. This hybrid approach allowed a theoretically-informed understanding or error reporting, but also the inclusion of unexpected experiences or influences associated with the complex primary care context.

The topic guide was reviewed for appropriateness by stakeholders with expertise in general practice, community pharmacy practice, primary care commissioning and behaviour change. We anticipated the topic guide would adapt as data collection and analysis progressed. The first interview was used to test the guide. Further to discussion and review by $\mathrm{NH}$ and GD, it was agreed no major changes were required.

Interviews were conducted by a female qualitative researcher $(\mathrm{NH}, \mathrm{PhD})$ with an academic background in psychology and health services research. NH had no prior knowledge of the participants or their roles in error reporting.

\section{Data analysis}

All interviews were audio recorded, transcribed verbatim and the transcripts anonymised. We continued interviewing until it was deemed that no new themes were being generated from additional interviews. Data collection and initial analysis occurred iteratively.

Framework analysis ${ }^{35}$ was used for coding and charting the qualitative data with the help of QSR International's NVivo software (V.12, 2018). After familiarisation with the data, a hybrid approach to thematic analysis using both deductive and inductive coding and categorisation was used to support the development of an initial analytical framework. This was informed, but not restricted by, constructs from the COM-B model: psychological and physical skills, ability and knowledge (capability); reflective and automatic processes directing decisionmaking and behaviours (motivation); and social and environmental factors that make behaviour possible (opportunity).

A selection of initial transcripts were dual coded by $\mathrm{NH}$ and GD to test coding agreement and the use of the framework. Minor amendments were made accordingly. All transcripts were then coded using the refined framework. Categories of data from each transcript, including references to illustrative quotations, were charted into a framework matrix to allow comparisons to be made across and within accounts using constant comparison. Final interpretation of the data analysis was refined further to discussion with the project steering group.

\section{Ethical and governance considerations}

Participants were asked not to provide identifiable details on specific cases. Anonymised data from interview transcripts has not been deposited to a publicly available data repository due to the potential inclusion of sensitive potentially identifiable information.

\section{Patient and public involvement}

Early feedback from members of the National Institute of Health Research Design Service North East and North Cumbria consumer panel was integrated into the initial stages of the study design and topic guide. Following their advice, patients and the public will be consulted to ensure appropriate public engagement with the study findings.

\section{FINDINGS}

We interviewed 25 participants from a range of organisation types, the majority of whom had dual roles. This allowed the exploration of a variety of information rich perspectives (see table 1). Interviews ranged from 39 to 70 min (mean $52 \mathrm{~min}$ ).

\section{Accounts of reporting behaviour}

All interviewees provided examples of prescribing errors they had encountered. Participant accounts highlighted a complex infrastructure of reporting and feedback systems across primary care and variability in reporting practices.

If I was being honest, I would think that most prescribing errors are not reported at all... about $90 \%$ of significant events are reported by $10 \%$ of people in the practice. (general practitioner, 11)

Three of 11 prescribers had never reported an error or incident, 6 had used an internal significant event form or log, some of which may have been reported by administrative teams on local electronic reporting systems. The most commonly used local electronic reporting 
Table 1 Participant characteristics $(n=25)$

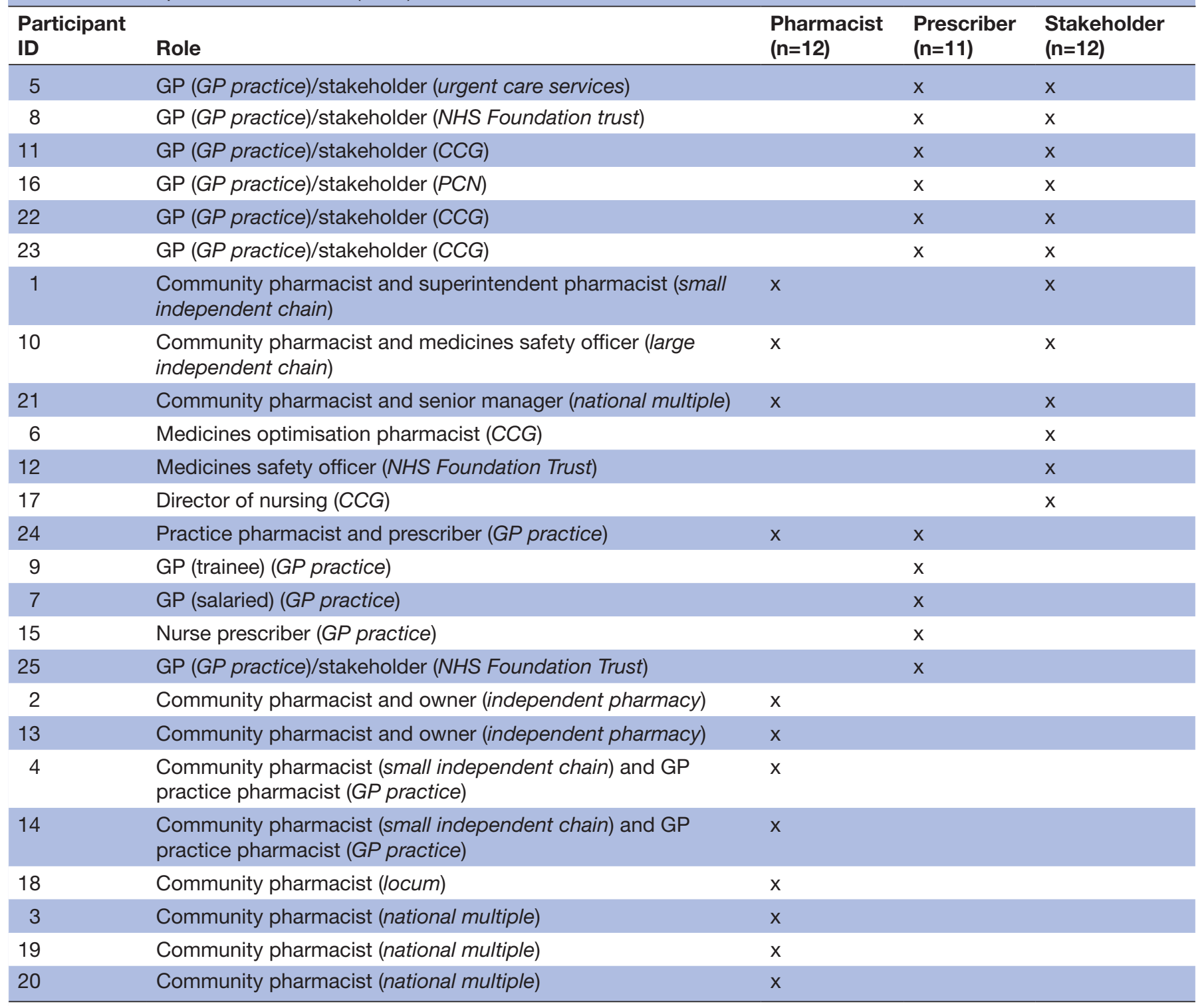

CCG, Clinical Commissioning Group; GP, general practitioner; NHS, National Health Service; PCN, Primary Care Network.

system by our participants was the 'significant incident reporting system', SIRMS, commissioned by local CCGs and supported by their regional commissioning support service. Two prescribers working in out of hours community care services had used the electronic reporting system available through their secondary care trust. Most pharmacists we interviewed from national multiple pharmacies used bespoke reporting systems within their organisations, but these were rarely used to report prescribing errors. Three of the 12 pharmacists never reported or recorded prescribing errors. Two reported that prescribing 'interventions' were recorded using internal paper-based systems. One of the 12 pharmacists (an owner of a sole pharmacy) had reported errors directly via England's national reporting system (NRLS) system and this had been less than four times over the last 5 years. One had reported electronically via the National
Pharmacy Association's online system. Rectifications made to prescriptions within community pharmacy were more usually recorded on internal patient medication record (PMR) systems, but not externally reported. Only one stakeholder referred to the national Strategic Executive Information System, which is a national system in England used to report serious incidents.

Although the main focus of our study was initially on reporting that feeds into formal infrastructures allowing the identification of error patterns to support future learning, findings identified that what is understood and described as 'reporting' can also vary. The term 'reporting' was used to reflect a range of different behaviours. As well as formally recording identified errors on an electronic or paper reporting system (internal or external to an organisation), reporting was also referred to as: advising or sharing concerns with a superior; informal logging or 


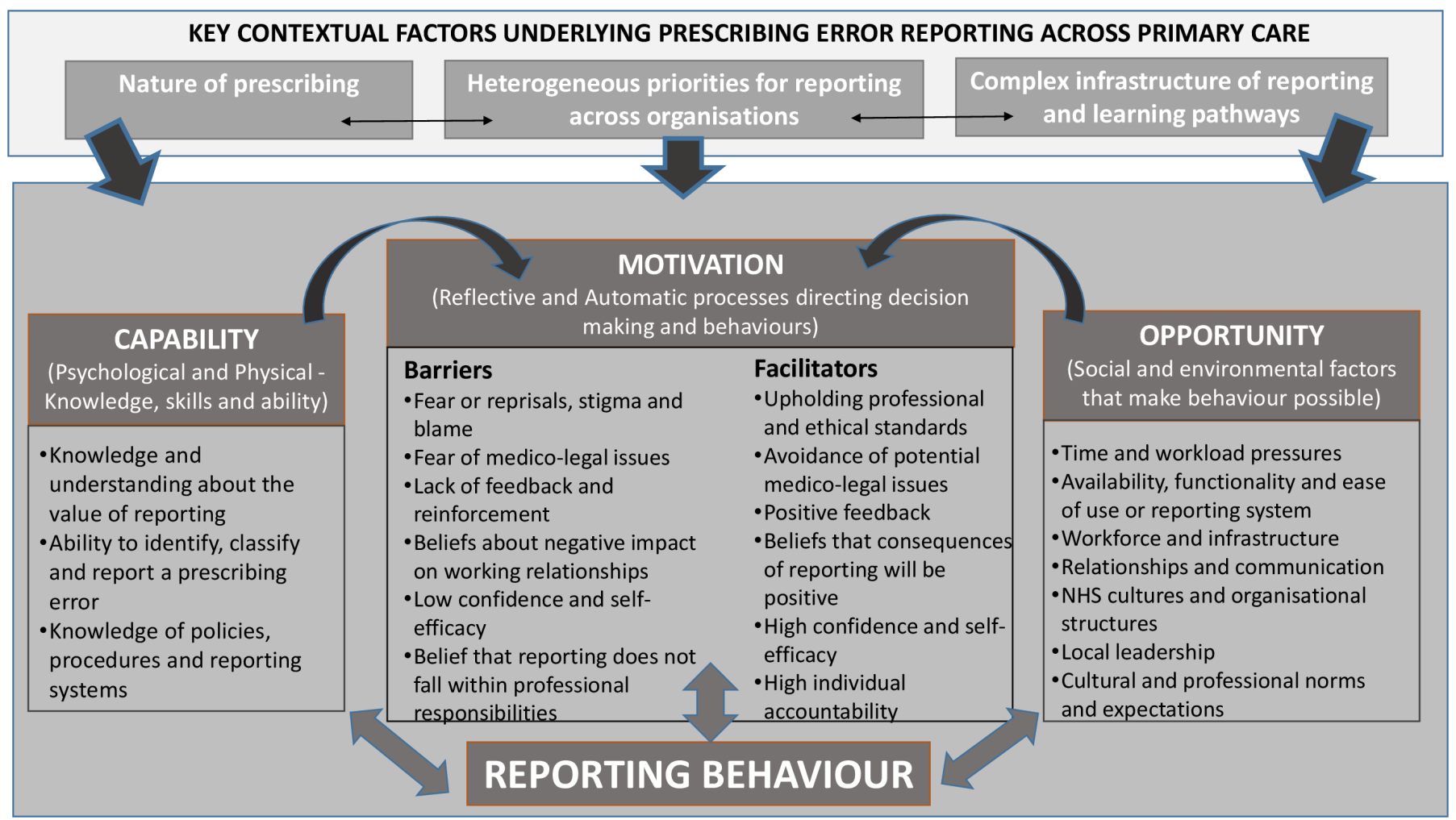

Figure 1 Mapping of influences on reporting to constructs from the COM-B model.

recording; and/or logging for inclusion in a significant event meeting. Prescribing errors were more likely to be resolved and logged internally rather than reported using a formal external reporting system, unless they were deemed to have caused significant harm, or had the potential to. When a prescribing error was deemed to be associated with a significant incident or event, formal reporting occurred more quickly and issues escalated directly to superiors or other colleagues. We therefore included within our analysis any reporting behaviour as defined by participants that occurred as a result of the identification of a prescribing error.

\section{Influences on prescribing error reporting behaviour}

Participants described a range of influences on their capability, opportunity and motivation to report a prescribing error. These were mapped to the constructs from the COM-B model and are summarised in figure 1.

Our analysis, however, also identified three cross-cutting themes that describe critical contextual features of particular significance to prescribing error reporting and the primary care setting. These were found to underpin influences on reporting behaviour mapped directly to COM-B constructs and reflect participant accounts around: the nature of prescribing; heterogeneous priorities for reporting across and within different primary care organisations; and the complex infrastructure of reporting and learning pathways across primary care. The proposed relationship between these contextual themes and those that mapped more directly to COM-B constructs is illustrated in figure 1 and is described in more detail below.

\section{Capability}

The ability to identify, classify and report a prescribing error, and knowing what prescribing errors to report and to where influenced reporting behaviour directly and also indirectly through the impact on motivation. There was unacknowledged contradiction within participants' accounts from all participant groups in relation to reported confidence in their ability to correctly identify and define a prescribing error and knowing which to report. On the one hand, and 'above the surface', this was described as straightforward, with clear consensus around the value of reporting in principle, and agreement about the definition of a prescribing error. This was particularly the case where there was strong shared agreement about the potential for the error to cause significant harm; those that were associated with 'wrong drug, wrong dose, wrong form, wrong person'; where selection of the drug was clearly 'not appropriate' for an individual patient (eg, known contraindications); and/or were deemed to be preventable. On the other hand, a range of complexities were apparent 'below the surface' associated with the ability to identify and define a prescribing error, interpret its significance and knowing when and where to report in keeping with expectations, reporting policies, guidelines and systems.

The nature of prescribing itself was described as playing a crucial role within this process. Prescribing relies on individual clinical judgement as well as prescribing guidelines and local formularies, which can change over time and across the primary care setting. Rather than 
consisting of defined cut-offs and absolute thresholds of correct or incorrect prescribing (errors), accounts reflected more of a continuum with definite easily identifiable, significant or 'true' prescribing errors at one end and suboptimal or more 'risky' prescribing at the other. The following quote from on GP exemplifies how types of errors could be differentiated.

a true error would be, say, to prescribe someone who's got a penicillin allergy, penicillin. Or a child, an adult dose of a medicine. But I guess what I'm including in, in, prescribing errors is when we prescribe an anti-inflammatory drug to someone who's got a peptic ulcer, and it could cause them to have a gastric bleed. (general practitioner, 23)

The language used by participants, for example, terms such as 'slip-ups' or 'inaccuracies', reflect these complexities, underlying beliefs about potential for harm, knowledge of different error types and thresholds for reporting, which all impact on the capability (and motivation) to report.

There's loads of things where some things are not completely right... Things that do not come to patient harm, but there are a lot of inaccuracies"... "it's not even easy to describe what is a clear prescribing error. (general practitioner, 7 )

Another GP described how standard reporting systems and structures did not always acknowledge the underlying complexity around prescribing safety and error reporting.

If you've just got someone ticking boxes then there's no proportionality because then it's either right or wrong and in prescribing it's often not like that. (general practitioner, 5)

The identification and classification of a prescribing error was therefore at times associated with a degree of ambiguity and temporal uncertainty, as well as variability in individual assessment, beliefs and perceptions about the potential for harm, the time point at which prescribing could be defined as an 'error' and the value of reporting. Additional examples provided by participants are provided in table 2 .

There was, in particular, a lack of consensus around when an error might be classified or defined as a 'near miss' and when and where these should be reported. Within the taxonomy used for the NRLS, a 'near miss' is defined as ' any patient safety incident that had the

Table 2 Illustrative examples of the ambiguity associated with the identification and definition of prescribing errors

\section{Example issues Illustrative quotes}

Correctly prescribed "so it's not necessarily a prescribing error on behalf of the GPs, but they might inadvertently prescribe based on information the wrong thing, because nobody's told them that they've changed what the patient's on. It's a vicarious available at time of prescribing error if you like, because they're prescribing what they believe is the right medication, ... prescribing. and you know it's not necessarily that the person is going to be harmed by that wrong medication because they were on it before, it's just they've changed onto either a different dose or um a different medication." (stakeholder, 17)

Differences in "But they can prescribe what they deem to be appropriate, which might not be what I consider to be professional opinion about appropriateness of prescribing. appropriate. ... So, sometimes we'll query the antibiotic for a one-and-a-half year old, and they'll say, 'it's only a soft indication.' I'm like, 'you shouldn't be prescribing for soft indication,' you know? And then I'm just like, well, I could. But then I'm within my right to say, 'well, I'm not giving it out.' But that doesn't help the patient. It doesn't really help- really help anybody. Erm, but yeah. So, sometimes I'm just like, 'oh [sighs]. I don't really think that's particularly appropriate but we would record it but we probably wouldn't report it." (pharmacist, 3)

Unavailable "what could be perceived, what could be a prescribing error for one individual may not be for another information on which individual so, its' all quite subjective. There're some clear ones that I used, for the example of to base an error judgement at the paracetamol, which is very clear cut but, even in some people where you couldn't take the normal dose dispensing stage. because it just wouldn't be appropriate for them, and just because of their metabolism and so on, but you would need more information, that wouldn't necessarily be visible. Summary care records have definitely helped." (pharmacist/stakeholder, 21)

Historically accepted "So, is that a drug error? is that an incident that should be reported? ... Now, we know increasingly prescribing based on guidance that is that that is not the right thing to be doing but of course there's many, many people taking those later found to cause patient harm.

Correct dose not clear until patient responds (or not) to medication. medications." (GP, 22)

GP, general practitioner.

"So, they can prescribe it [lower dose]. They're legally allowed to. It's not harmful to the child. It might just not be that effective. But if it treats it at that lower dose, which is what they think it will do, then fine. But if the infection isn't treated and the child requires more antibiotics, then that then means that the original issue was a prescribing error." (pharmacist, 3) 
Box 1 Illustrative quotes relating to variable definitions of a "near-miss"

"but if a pharmacist picks that up and intercepts that in the meantime, for me that's a near miss because it didn't become dangerous because the patient didn't get it... But that's just my interpretation." (nurse, 19) "I think most errors are a near miss .... anything which is either clinically inappropriate or there is an allergy contra-indication for why they should be prescribed that medication, or etcetera all of those things would come in to it including form, about giving a tablet to a 3-year-old as it really needs to be a liquid would be prescribing error in my book, but they can be categorised as you know, are they serious or not so they would all be near misses but near misses which would cause different levels of harm ... is how I would see it." (pharmacist/stakeholder, 21)

"So, I just see that as a system working effectively, ... if the pharmacist has the conversation that results in the change in the prescription. I think the errors come if it leaves the pharmacist and is with the patient." (general practitioner/stakeholder, 22)

potential to cause harm but was prevented, resulting in no harm to people receiving NHS-funded care' ${ }^{36}$ The ability to determine which prescribing events should be defined as 'near misses' and required reporting, however, could be influenced by expectations and cultural norms around prescribing practices, roles and accountability.

You could have practice nurses who are prescribers who would report everything because they are worried for their registration. They think I'd better report that because I don't want the comeback on me that I didn't report it. When really it might not need to be reported, if you know it's over reporting if you like. Whereas you might have some GPs who have done it for thirty odd years. They know what they're prescribing, they know best, that's not an incident, I didn't give it to the patient., so I think because there's that much variation in roles, responsibilities, accountabilities. (nurse prescriber, 15)

The 'near miss' definition also seemed to create challenges when applied in a cross-organisational context, and this can be seen using examples from our participants in box 1 .

These examples demonstrate how the definition of a as 'near miss' can depend on: whether a medicine had been dispensed or reached the patient, whether the incident had resulted in harm, the perceived severity of potential harm, beliefs about professional responsibilities and accountability for prescribing and dispensing, and, whether the prescription had been contained within organisational boundaries. Complexity around the ability to identify and define an error plays a key role in reporting behaviour directly as well as indirectly on the motivation to report. It also impacts on views about the perceived frequency of prescribing errors in primary care, (which varied across the interviews from 'almost never' to 'routinely'), and decisions about whether and when to inform patients.

\section{Opportunity}

Interviews highlighted a number of influences on the social and physical opportunity to report (see figure 1 ). These opportunities varied across the primary care setting depending on heterogeneous professional and organisational norms, expectations and priorities as well as the complex infrastructures and systems with different functionalities to support error reporting already described above.

A commonly reported opportunity barrier was the lack of time associated with workload and staffing pressures, compounded by time consuming reporting processes. This can influence reporting behaviour directly, but also could influenced motivation to report if the significance of the potential harm was perceived to be low and the potential for wider learning was not felt to be outweighed by the time and effort required to go through the reporting process.

the reporting mechanisms are you know, they're all big forms and they're... you know, you need time to sit down and go through them and remember and have the notes and remember all the things that have happened and they are time consuming. Often, it's the big ones that you feel so shocked about that you will sit down and report ...there's so many pulls on your time, it sometimes has to be quite a significant, serious thing to then make you sit down and do it. (general practitioner, 9)

Social opportunity to report was felt to be supported across all settings by strong local leadership, facilitating an open culture of reporting. This helped to increase motivation to report and was also seen to be important in helping to counter perceptions of what was described as a 'blame culture' within the NHS more widely. Relationships and hierarchies within organisations and between professionals groups could, nevertheless, also have negative effects on the opportunities as well as motivation to report. The relationship between the dispenser and prescriber, in particular, was a commonly reported influence on the opportunity to identify and motivation to report prescribing errors.

there has definitely been a shift in that culture, but I think again a pharmacist is still very mindful not to undermine that relationship [with the GP] but will be more open with I do need to check this dose to make sure it is appropriate for you [the patient]... there will be a little bit of risk of am I going to upset my GP and is he then going to say he sends all his scripts over there. It's down to the professionalism ... I think it would be erm, remiss of me to pretend that doesn't influence people. (community pharmacist and senior manager, 21)

Functionality of reporting systems also influenced reporting behaviour directly as well as indirectly through the influence on motivation. Within community pharmacy, for example, the functionality within bespoke 
organisationally tailored reporting systems, was reported to not always reflect the potential overlap between a prescribing and dispensing error, as exemplified by the following quote by one pharmacist with a national safety role.

our system isn't intuitive. It doesn't really direct, it doesn't ask them to put them [prescribing errors] on. (community pharmacist, 21)

\section{Motivation}

Motivation to report was influenced by capability and opportunity as described above. Other barriers and facilitators to motivation were also described and are summarised in figure 1 . These influences on motivation were also clearly impacted by the nature of prescribing, heterogeneous priorities for reporting evident across primary care organisations and complex reporting and learning pathways.

For example, although the value of error reporting in principle was clearly recognised across all participant groups, some of the GPs we spoke to were less motivated to report all prescribing errors as they placed higher value on other safety improvement initiatives for improving prescribing quality, such as medicines optimisation initiatives and audits, aimed at identifying and addressing 'sub-optimal' or 'risky' prescribing. Perceived consequences and value of reporting in relation to other initiatives seems to be underpinned by their views about the nature of prescribing more generally and the emphasis on learning from the SEA process within their practices. Accounts suggest that within general practice, motivation for reporting outside of the organisation via electronic reporting systems (eg, SIRMS) was increased for events that were judged as having resulted in significant patient harm, and which have usually been included within their SEA processes, as per their contractual requirements. Prescribing errors were reported as rarely included in this category and tended not to be formally reported inside or outside of the organisation.

In terms of filling the forms and reporting, it just doesn't happen very much and that is the culture which lots of GP surgeries are living in. (general practitioner, 7)

However, variability between and even within practices was reported.

it's variable between the practices.... most practices will have an internal system for recording errors. But won't share it externally by putting it onto SIRMS. (general practitioner, 23)

Within community nursing, out of hours services and care home settings, community based nursing and medical staff were in a position to be able to detect and report on their own prescribing and administration errors, as well as those of other prescribers from other parts of the healthcare system. Due to commissioning arrangements, staff from this setting had access to a complex number of different formal and informal reporting systems and processes depending on the source and type of error that was identified with the potential of needing to navigate between different reporting processes, expectations and priorities.

All participant groups highly valued the role of community pharmacists in identifying and rectifying prescribing errors in line with national regulatory and contractual requirements. When 'prescribing errors' were identified within community pharmacy, however, these were more commonly 'flagged', 'investigated', 'rectified" or "interventioned" rather than being formally reported or even recorded.

what we do is we identify [the error], rectify it and we move on... and we're not very good I don't think always recording them. (community pharmacist, 3)

When they were recorded, this was usually on the internal PMR rather than other internal or external formal or informal error reporting systems. Motivation to record included ensuring individual patients received appropriate medications in the future and medicolegal concerns in the event of a prescribing decision being investigated or identified as a dispensing 'error' or the responsibility of the pharmacist at a later date.

So, it would be also cover yourself, I guess, as a pharmacist, to say, 'I did query this. The child is still unwell.' So, then if the parent or whoever turns around and says... or goes to another prescriber who's like, 'this was really inappropriate' and then they report it, and then someone comes back to me and says, "why did you not report it?' I'll say, 'well, I did make notes and then whenever I queried it again, they changed it.... (community pharmacist, 3 )

Within community pharmacy, low reported levels of prescribing error reporting seemed to be in sharp contrast with an evident and established culture and acceptance of reporting 'dispensing errors and near misses'. This distinction seemed to be inextricably tied to notions of professional accountability, as well as the desire to avoid any negative impact on established relationships with prescribers. Many pharmacists described 'assuming', 'hoping' or 'expecting' prescribing errors to be reported by the prescriber.

I report ours [dispensing errors] that we make in the pharmacy... But I don't tend to report the doctors' [prescribing] ones. When we report it back to the doctor, they should be reporting it themselves... Either, kind of like, a significant incident or a SIRMS, it'd be on their kind of monitoring system. (community pharmacist, 4)

Prescribing errors that originate in one primary care organisation but are identified in another, (such as community pharmacy) particularly those that do not 
reach the patient, do not therefore seem to be routinely externally reported.

Well, the ones [prescribing errors] that are picked up by community pharmacists, er ... on a routine basis, I don't think are reported anywhere. (general practitioner, 16)

Due to community pharmacists role in the identification of prescribing errors, there was some acknowledgement across all settings of currently untapped opportunities for supporting the optimisation of prescribing error reporting and thereby the identification of error trends that could help improve patient safety. Some key practical challenges and barriers to the opportunity and motivation to report were also highlighted. These included: workload pressures and time burden; the need for a reporting system that was in line with the rest of the primary care community to enable reports to be submitted collectively with the prescriber if appropriate; and addressing interprofessional relationships between prescribers and community pharmacy. For example, it was highlighted by one GP that shifting perceptions of the role of community pharmacist from 'valued safety net' to 'safety assessor' could have a potential detrimental impact on relationships and communication.

I think that changing that from being a safety net, which is part of the community pharmacist's perceived role, and actually value added, to actually then being more of an assessment of safety, I think would change the dynamics. (general practitioner, 8)

\section{Feedback and learning}

Feedback and learning from previous reporting could also help reinforce motivation to report. Examples were provided of feedback systems and local information sharing through patient safety groups and committees and other communication networks that were seen generally to have a positive impact on learning at a local level and improving patient safety. Learning events resulting from prescribing issues identified formally or informally were also reported. Involvement of community pharmacists in team practice meetings to discuss and share learning from medication errors was reported to be infrequent but valued when it occurred. There was also a perceived need to improve feedback and learning across the primary and secondary care interface to support prescribing quality improvements.

Motivation to report could be negatively influenced by a perceived lack of feedback on reports submitted and beliefs about the lack of consequences of reporting on future learning, harm prevention or quality improvement. Many interviewees were not aware of any routine feedback and felt that when aggregated feedback on error reporting was provided, this did not include enough information relevant to prescribing in primary care. Because the level, quality and quantity of data recorded varies across settings and commissioning organisations, capturing a true picture with the available data on which to base learning needs was not always felt to be possible.

there isn't any way of identifying with any level of accuracy what the true rate of prescribing errors is... in one place... because it's reported in different places nobody's got that oversight of everything, nobody is able to put it all together in one place. (stakeholder, 17)

\section{DISCUSSION}

This study provides a novel in-depth whole system exploration of the facilitators and barriers to medication prescribing error reporting behaviour across primary care organisations in the UK. Our analysis draws attention to some key challenges in this area, including inconsistent and low levels of prescribing error reporting across primary care organisations, a lack of shared definitions and understanding about which prescribing errors should be reported by whom and to where, and a complex infrastructure of reporting systems and feedback pathways across different provider and commissioning organisations. Our data confirms that inconsistencies in system use, priorities and processes across primary care in relation to prescribing error reporting is likely to be creating an evidence gap, which may be limiting the identification of wider trends and learning potential to optimise patient safety.

Despite evident widespread recognition of the value of error reporting in principle this does not seem to be sufficient to facilitate consistent and high levels of prescribing error reporting across primary care organisations. In line with components of the COM-B model, capability, opportunity and motivation to report were found to be strongly influenced by organisation context and resources, the wider primary care setting as well as more entrenched views about professional roles and responsibilities. Our findings thereby concur with previous evidence around the impact of wider and complex sociocultural, organisational and political processes and agendas relating to professional practice, regulation, social inequalities, control and power. ${ }^{27-29}$ A climate of fear within the NHS ${ }^{37}$ and need for trust of and understanding by regulatory authorities ${ }^{38}$ have previously been described and were also mentioned as influences on reporting by our participants. Beliefs around the consequences of reporting included, in particular, sensitivities around interprofessional relationships with colleagues or prescribers, which have also been highlighted in other settings and error reporting contexts. 3940

Community pharmacy is seen to provide a valued and cost-effective safety net within primary care for minimising harm from prescribing errors. ${ }^{21}$ It is estimated that around $1 \%-2 \%$ of all prescriptions are checked with the prescriber. ${ }^{41}$ There was acknowledged potential for an increased role for community pharmacy in optimising prescribing error reporting, but also a reluctance to shift 
perceptions of the role of community pharmacist from 'valued safety net' to 'safety assessor' . Underlying causes of prescribing errors are complex ${ }^{42}$ and concerns were raised by pharmacists and prescribers that pharmacists may not always have sufficient knowledge of the patient, their medical history or the prescribing incident to be able to correctly identify, classify or report a prescribing error. Although templates are available for community pharmacies to record safety incidents from external organisations, formal reporting of these incidents was not reported as routine by our community pharmacist participants, in line with findings by Ashcroft, et al... ${ }^{43}$ Our findings demonstrate how motivation to report prescribing errors is more likely in this setting if there is an element of dispensing related accountability apportioned to the pharmacist.

From a systems or human factors approach, patient safety, quality and errors can be viewed as 'emergent properties' of the interaction between people and the system they work in. ${ }^{11}$ Prescribing error reporting takes place within an interconnected, and exceptionally stretched, primary care system that interfaces closely with secondary care as well as independent organisations with commercial interests. Current increasing demands within primary care ${ }^{44}{ }^{45}$ can contribute to conflicting priorities with clinical workflow, increased levels of patient safety incidents ${ }^{4647}$ and a reduction in prescribing error reporting. ${ }^{1148-50}$

Workload and time pressures can be compounded by barriers associated with reporting systems and processes ${ }^{32}$ which were described as burdensome and time consuming and impacted on both the opportunity and capability to report across primary care. In line with other research, our findings suggest that opportunity to report can be influenced by awareness and functionality or reporting systems ${ }^{23}{ }^{24}$ a lack of clear reporting criteria, ${ }^{25}$ availability of reporting mechanisms that fit around daily work$\operatorname{load}^{244851}$ and require minimum effort and time. ${ }^{30}$

Our findings also concur with previous evidence that under-reporting can be associated with a lack of standardised definition of a prescribing error or incident, ${ }^{1751-54}$ poor knowledge of what types of prescribing errors need to be reported and to whom ${ }^{11} 505556$; and a lack of clarity over roles and responsibilities. ${ }^{57}$ Prescribing errors have been helpfully defined by Dean, et $a \bar{l}^{4}$ as 'unintentional significant reduction in the probability of treatment being timely and effective or increase in the risk of harm when compared with generally accepted practice'. However, in line with qualitative interview findings around safe care more generally, ${ }^{47}$ our findings highlight that the way in which researchers and policymakers define 'prescribing errors' do not always have practical meaning for frontline staff and this can inhibit reporting. In practice, classifying prescribing as a reportable error involves a high level of individual interpretation and complex assessment around the perceived significance of actual or potential harm, ${ }^{50}$ what is generally accepted practice (which can vary over time and place), perceived frequency and significance of the error type.$^{30}$ Prescribing events classified as 'near-misses', which varies depending on organisational boundaries, the point at which they were corrected, and whether they reached the patient or resulted in harm, were unlikely to be internally or externally reported in any primary care setting.

The new PSIMS which will replace the current reporting system (NRLS) in England includes a new taxonomy and has beneficially moved to the use of the less judgemental term 'events' rather than 'errors'. ${ }^{9}$ How taxonomies within this and other reporting systems are used and interpreted has not been explored. Another key change will be that records will be amendable, which means issues around the identification and definition of prescribing as an 'error' or 'near miss' changing over time will be addressable in the new system. There is, nevertheless, a need to also improve functionality of the systems that feed into the PSIMS to ensure they are less burdensome and time consuming to complete and that they incorporate categorisation that meets the needs of all users, providers and commissioners.

A number of trials and evaluations have shown that interventions can reduce prescribing defined as 'highrisk', ${ }^{58}$ and improve prescribing systems. ${ }^{53159}$ Prescribing defined as high risk, which is common in primary care, ${ }^{60}$ may not always be inappropriate however ${ }^{58}$ and along with suboptimal prescribing may or may not be interpreted as a reportable prescribing error. Participant accounts highlighted a desire for proportionality and an underlying pragmatic judgement and balancing of the barriers and facilitators implicit within the decision to report individual incidents that vary across organisations and professional groups.

As previously identified by Mitchell, et $a l^{57}$ perceived lack of feedback and learning potential of reporting was another key influence on future motivation to report. Our findings suggest that most prescribing error reporting in primary care takes place via local risk management systems which vary across organisations and is associated with an exceptionally complex infrastructure of reporting and feedback pathways across different provider and commissioning organisations. Associated learning can occur without formal reporting, but this tends to be escalated on a more ad-hoc basis.

Key strengths of our study include: a varied purposive sample with information rich perspectives from prescribers, pharmacists and other key stakeholders, some of whom held additional national roles relating to prescribing primary care, commissioning, medicines safety and optimisation; we were able to continue interviewing until saturation of themes had been achieved; and data collection and analysis were grounded within an established theoretical framework of behaviour, the COM-B model. ${ }^{34}$ The latter allowed a theoretically informed lens through which to explore barriers and facilitators to reporting behaviour that would be important to understand when developing more effective and theory-informed solutions to facilitating changes in error 
reporting behaviour. We were able to identify a range of key influences on reporting behaviour that mapped directly to the constructs from the COM-B model (capability, opportunity and motivation). We chose, however, to also include inductive coding and encouragement of participant-led topics within the interviews. This enabled a focus on three key cross-cutting themes pertaining to the multiorganisational primary care context, including the nature of prescribing and heterogeneous priorities and reporting and learning infrastructure and processes, which we propose underpin many of the influences that map across the constructs from COM-B. Although COM-B recognises the influence of social and environmental factors on the opportunities to enact a behaviour, our findings align with existing debate around the value of drawing on theories of behaviour that incorporate social, cultural and economic contextual factors as well as a focus on individual influences when developing behaviour change interventions. ${ }^{61}$

There are limitations to our study, however. Data were collected across a small region in North East England, and although some participants were able to provide wider and even national perspectives, this may limit transferability to other contexts and regions, particularly due to the complexity and varying infrastructures around error reporting and learning. Furthermore, we were not able to include perspectives from relevant regulatory bodies or from prescribing staff from community nursing and dentists. Although our data includes accounts from prescribers and pharmacists who were able to describe their decisions not to report identified prescribing errors, there may be a degree of bias as those who agreed to be interviewed may have been likely to have a specific interest in and positive attitudes towards the value of prescribing error reporting more generally.

\section{CONCLUSIONS}

A theoretically informed understanding of the influences on reporting behaviour can help to identify strategies to optimise reporting and reporting systems. It can also provide insight into the potential limitations associated with the analysis of reported events and subsequent potential for learning and harm prevention. Our findings highlight how the capability, opportunity and motivation to report prescribing errors in primary care is influenced by a range of individual, organisational and wider socio-cultural factors . Key challenges include heterogeneous priorities for reporting across and within different primary care organisations, the nature of prescribing itself and the complex infrastructure of reporting and learning pathways.

A clearer consensus is needed to help optimise prescribing error reporting and identify more systematic and efficient cross-organisational solutions to preventing medication-related harm across primary care in a way that builds on existing relationships and communication, is sensitive to important differences across organisations and professional groups and considers an acknowledged potential for an increased role of community pharmacy. Findings may also help support the more effective use of new technologies for error reporting currently being developed by the NHS.

Correction notice This article has been corrected since it was published. The name of the funder has been corrected.

Acknowledgements We would like to acknowledge the Academic Health Science Network, North East and North Cumbria for funding the study (grant number PSC Q19). Thanks are due to all the collaborators (Alastair Buxton, Pharmaceutical Services Negotiating Committee, England; Janice Perkins, Chair, Community Pharmacy Safety Group; Ann Fox, Director of Nursing, Quality and Safety, Sunderland CCG) for their assistance with participant recruitment and their appreciated input in the development of the proposal and comments and feedback on study oversight. We would also like to thank Lucie Mussett from NHS Improvement for her early input into the project. Particular thanks are due however to all the participants who kindly shared their valuable time and views with us. This work would not have been possible without their helpful and insightful input.

Contributors GD was responsible for the initial conception of the study. GD and $\mathrm{NH}$ led on the study design with substantial contributions from KB, JS, NW and SW. NH completed the data collection and analysis with contribution from GD. All authors contributed to interpretation of data. GD, KB, JS, NW and SW provided essential input around the interpretation of findings in relation to the wider primary care, pharmacy and medicines safety context. NH accepts full responsibility for the work and the conduct of the study, had access to the data, and controlled the decision to publish. NH drafted the manuscript and all authors critically revised drafts for important intellectual content. All authors have approved the final version of the manuscript. All authors agree to be accountable for all aspects of the work in ensuring that questions related to the accuracy or integrity of any part of the work are appropriately investigated and resolved.

Funding This work was supported by the Academic Health Science Network, North East and North Cumbria, grant number PSC Q19.

\section{Competing interests None declared.}

Patient and public involvement Patients and/or the public were involved in the design, or conduct, or reporting, or dissemination plans of this research. Refer to the Methods section for further details.

Patient consent for publication Not applicable.

Ethics approval Approval was granted by Sunderland University Research Ethics Group. There is no reference number. Participants gave informed consent to participate in the study before taking part.

Provenance and peer review Not commissioned; externally peer reviewed.

Data availability statement Data are available upon reasonable request. Anonymised interview data has not been shared to a repository to protect the anonymity of participants who could be identified by their unique roles in their organisations. The authors are prepared to consider requests for appropriately managed data sharing on an individual case by case basis.

Open access This is an open access article distributed in accordance with the Creative Commons Attribution Non Commercial (CC BY-NC 4.0) license, which permits others to distribute, remix, adapt, build upon this work non-commercially, and license their derivative works on different terms, provided the original work is properly cited, appropriate credit is given, any changes made indicated, and the use is non-commercial. See: http://creativecommons.org/licenses/by-nc/4.0/.

ORCID iD

Nicola Hall http://orcid.org/0000-0002-0216-512X

\section{REFERENCES}

1 Garfield S, Barber N, Walley P, et al. Quality of medication use in primary care--mapping the problem, working to a solution: a systematic review of the literature. BMC Med 2009;7:50.

2 World Health Organisation. The third who global patient safety challenge: medication without harm, 2018. Available: https://www. who.int/patientsafety/medication-safety/en/ [Accessed 10 Dec 2018]. 
3 Donaldson LJ, Kelley ET, Dhingra-Kumar N, et al. Medication without harm: who's third global patient safety challenge. Lancet 2017;389:1680-1.

4 NHS England. Next steps on the NHS five year forward view, 2017. Available: https://www.england.nhs.uk/five-year-forward-view/nextsteps-on-the-nhs-five-year-forward-view/patient-safety/ [Accessed 16 April 2018]

5 Elliott RA, Putman KD, Franklin M, et al. Cost effectiveness of a pharmacist-led information technology intervention for reducing rates of clinically important errors in medicines management in general practices (pincer). Pharmacoeconomics 2014;32:573-90.

6 Kohn L. To err is human: an interview with the Institute of medicine's Linda Kohn. Jt Comm J Qual Improv 2000;26:227-34.

7 Department of Health. Expert group on learning from adverse events in the NHS: an organisation with a memory. London, 2000.

8 Cousins D, Gerrett D, Richards N, et al. Initiatives to identify and mitigate medication errors in England. Drug Saf 2015;38:349-57.

9 NHS England. Patient safety incident management system, 2020. Available: https://www.england.nhs.uk/patient-safety/patient-safetyincident-management-system/ [Accessed 01 Oct 2020].

10 Improvement $\mathrm{N}$. The future of the patient safety incident reporting: upgrading the NRLS, 2017. Available: https://improvement.nhs.uk/ news-alerts/development-patient-safety-incident-managementsystem-dpsims/ [Accessed 16 April 2018].

11 de Wet C, Bradley N, Bowie P. Significant event analysis: a comparative study of knowledge, process and attitudes in primary care. J Eval Clin Pract 2011;17:1207-15.

12 Care Quality Commission. Learning when things go wrong (healthcare services), 2017. Available: https://www.cqc.org.uk/ guidance-providers/healthcare/learning-when-things-go-wronghealthcare-services [Accessed 1 Aug 2020].

13 Cousins DH, Gerrett D, Warner B. A review of medication incidents reported to the National reporting and learning system in England and Wales over 6 years (2005-2010). Br J Clin Pharmacol 2012;74:597-604.

14 Panesar SS, deSilva D, Carson-Stevens A, et al. How safe is primary care? A systematic review. BMJ Qual Saf 2016;25:544-53.

15 Gaal S, Verstappen W, Wensing M. Patient safety in primary care: a survey of general practitioners in the Netherlands. BMC Health Serv Res 2010;10:21.

16 Kuo GM, Phillips RL, Graham D, et al. Medication errors reported by US family physicians and their office staff. Qual Saf Health Care 2008;17:286-90.

17 Avery AJ, Ghaleb M, Barber N, et al. The prevalence and nature of prescribing and monitoring errors in English general practice: a retrospective case note review. Br J Gen Pract 2013;63:e543-53.

18 Bullen K, Hall N, Sherwood J, et al. Prescribing error reporting in primary care: a narrative synthesis systematic review. Integrated Healthcare Journal 2020;2:e000026.

19 Chen Y-F, Neil KE, Avery AJ, et al. Prescribing errors and other problems reported by community pharmacists. Ther Clin Risk Manag 2005;1:333-42

20 Pharmaceutical Services Negotiating Committee Psirhpouc-ie-s-cgp-s-i-r. Patient safety incident reporting, 2013. Available: https:// psnc.org.uk/contract-it/essential-service-clinical-governance/patientsafety-incident-reporting [Accessed 01 Sep 2019].

21 Price Waterhouse Coopers. The value of community pharmacy detailed report, 2016. Available: https://psnc.org.uk/wp-content/ uploads/2016/09/The-value-of-community-pharmacy-detailedreport.pdf [Accessed 16 April 2018].

22 NHS England and NHS Improvement. Nhs oversight framework 2019/20, 2019. Available: https://www.england.nhs.uk/wp-content/ uploads/2019/10/nhs-oversight-framework-rev-oct19.pdf [Accessed 01 Aug 2020].

23 Kunac DL, Tatley MV, Seddon ME. A new web-based Medication Error Reporting Programme (MERP) to supplement pharmacovigilance in New Zealand--findings from a pilot study in primary care. N Z Med J 2014;127:69-81.

24 Kennedy AG, Littenberg B. Medication error reporting by community pharmacists in Vermont. J Am Pharm Assoc 2004;44:434-8.

25 Stavropoulou C, Doherty C, Tosey P. How effective are IncidentReporting systems for improving patient safety? A systematic literature review. Milbank Q 2015;93:826-66.

26 Waring J. Adaptive regulation or governmentality: patient safety and the changing regulation of medicine. Sociol Health IIIn 2007;29:163-79.

27 Waring J, Allen D, Braithwaite J, et al. Healthcare quality and safety: a review of policy, practice and research. Sociol Health IIIn 2016;38:198-215.

28 Waring JJ. Constructing and re-constructing narratives of patient safety. Soc Sci Med 2009;69:1722-31.
29 Keefer P, Kidwell K, Lengyel C, et al. Variability in threshold for medication error reporting between physicians, nurses, pharmacists, and families. Curr Drug Saf 2017;12:187-92.

30 Williams SD, Phipps DL, Ashcroft DM. Understanding the attitudes of hospital pharmacists to reporting medication incidents: a qualitative study. Res Social Adm Pharm 2013;9:80-9.

31 Avery AJ, Rodgers S, Cantrill JA, et al. A pharmacist-led information technology intervention for medication errors (pincer): a multicentre, cluster randomised, controlled trial and cost-effectiveness analysis. Lancet 2012;379:1310-9.

32 Fernald DH, Pace WD, Harris DM, et al. Event reporting to a primary care patient safety reporting system: a report from the ASIPS collaborative. Ann Fam Med 2004;2:327-32.

33 Verbakel NJ, Langelaan M, Verheij TJM, et al. Improving patient safety culture in primary care: a systematic review. J Patient Saf 2016;12:152-8.

34 Michie S, van Stralen MM, West R. The behaviour change wheel: a new method for characterising and designing behaviour change interventions. Implement Sci 2011;6:42.

35 Ritchie J, Spencer L. Qualitative data analysis for applied policy research. In: Bryman A, Burgess RG, eds. Analyzing qualitative data, 1994: 173-94.

36 National Patient Safety Agency. Seven steps to patient safety, 2004. Available: www.nrls.nhs.uk/sevensteps [Accessed 01 Aug 2019].

37 Berwick DM. The toxic politics of health care. JAMA 2013;310:1921-2.

38 Morgan L, Benson D, McCulloch P. Will human factors restore faith in the GMC? BMJ 2019;364:11037.

39 Phipps DL, Noyce PR, Parker D, et al. Medication safety in community pharmacy: a qualitative study of the sociotechnical context. BMC Health Serv Res 2009;9:158.

40 Rubin MA, Friedman DI. The ethics of disclosing another physician's medical error. Continuum 2015;21:1146-9.

41 Westein MP, Herings RM, Leufkens HG. Determinants of pharmacists' interventions linked to prescription processing. Pharm World Sci 2001;23:98-101.

42 Slight SP, Howard R, Ghaleb M, et al. The causes of prescribing errors in English general practices: a qualitative study. $\mathrm{Br} J$ Gen Pract 2013;63:e713-20.

43 Ashcroft DM, Morecroft C, Parker D, et al. Likelihood of reporting adverse events in community pharmacy: an experimental study. Qual Saf Health Care 2006;15:48-52.

44 Foundation PC. Making time in general practice, 2015. Available: https://www.primarycarefoundation.co.uk/images/PrimaryCareFound ation/Downloading_Reports/PCF_Press_Releases/Making-Time-in_ General_Practice_FULL_REPORT_28_10_15.pdf [Accessed 02 Dec 2019].

45 Society. NAaRP. Pharmacists and general practice: a practical and timely part of solving the primary care workload and workforce crisis, 2014. Available: https://www.rpharms.com/Portals/0/ RPS\%20document\%20library/Open\%20access/Publications/ Pharmacists\%20and\%20general\%20practice\%20-\%20RPS\% 20NHS\%20Alliance.pdf [Accessed 1 Nov 2019].

46 Hall LH, Johnson J, Watt I, et al. Association of GP wellbeing and burnout with patient safety in UK primary care: a cross-sectional survey. Br J Gen Pract 2019;69:e507-14.

47 de Wet C, Bowie P, O'Donnell C. 'The big buzz': a qualitative study of how safe care is perceived, understood and improved in general practice. BMC Fam Pract 2018;19:83.

48 Hickner J, Zafar A, Kuo GM, et al. Field test results of a new ambulatory care Medication Error and Adverse Drug Event Reporting System--MEADERS. Ann Fam Med 2010;8:517-25.

49 West DR, Pace WD, Dickinson LM. Relationship Between Patient Harm and Reported Medical Errors in Primary Care: A Report from the ASIPS Collaborative. In: Henriksen K, Battles JB, Keyes MA, eds. Advances in patient safety: new directions and alternative approaches (vol 1: assessment. Rockville (MD, 2008.

50 Rea D, Griffiths S. Patient safety in primary care: incident reporting and significant event reviews in British general practice. Health Soc Care Community 2016;24:411-9.

51 Rubin G, George A, Chinn DJ, et al. Errors in general practice: development of an error classification and pilot study of a method for detecting errors. Qual Saf Health Care 2003;12:443-7.

52 Rubin G. Medical errors. Terminology of "error" is important. BMJ 2001;322:1422.

53 Dean B. Learning from prescribing errors. Qual Saf Health Care 2002;11:258-60.

54 Dean B, Barber N, Schachter M. What is a prescribing error? Qual Health Care 2000;9:232-7.

55 Dovey SM, Phillips RL. What should we report to medical error reporting systems? Qual Saf Health Care 2004;13:322-3. 
56 Kennedy AG, Aiken CA. A survey of the medication error experiences of pharmacy technicians. Journal of Pharmacy Technology 2006;22:42-7.

57 Mitchell I, Schuster A, Smith K, et al. Patient safety incident reporting: a qualitative study of thoughts and perceptions of experts 15 years after 'To Err is Human'. BMJ Qual Saf 2016;25:92-9.

58 Guthrie B, Kavanagh K, Robertson C, et al. Data feedback and behavioural change intervention to improve primary care prescribing safety (EFIPPS): multicentre, three arm, cluster randomised controlled trial. BMJ 2016;354:i4079.
59 MacBride-Stewart S, Marwick C, Houston N, et al. Evaluation of a complex intervention to improve primary care prescribing: a phase IV segmented regression interrupted time series analysis. $\mathrm{Br} J \mathrm{Gen}$ Pract 2017:67:e352-60.

60 Stocks SJ, Kontopantelis E, Akbarov A, et al. Examining variations in prescribing safety in UK general practice: cross sectional study using the clinical practice research Datalink. BMJ 2015;351:h5501.

61 Davis R, Campbell R, Hildon Z, et al. Theories of behaviour and behaviour change across the social and behavioural sciences: a scoping review. Health Psychol Rev 2015;9:323-44. 